\title{
Gestión pedagógica docente y ejecución instrumental en estudiantes de una Escuela Superior de Formación Artística
}

\section{Teaching pedagogical management and instrumental execution in students of a Higher School of Artistic Training}

\section{Edwin De La Cruz Bautista}

Universidad Nacional Mayor de San Marcos, Lima, Perú.

\author{
Recibido 18-05-17 \\ Aprobado 02-08-17 \\ En Línea 10-08-17
}

\begin{tabular}{lll} 
Correspondencia & & Citar como: \\
${$\cline { 1 - 2 }$} }$ & & $\begin{array}{l}\text { De la Cruz, E. (2017). Gestión pedagógica docente } \\
\text { y ejecución instrumental en estudiantes de una }\end{array}$ \\
& $\begin{array}{l}\text { Escuela Superior de Formación Artística. Propósitos } \\
y \text { Representaciones, 5(2), 321 - 357. doi: http:// } \\
\text { dx.doi.org/10.20511/pyr2017.v5n2.175 }\end{array}$ \\
\hline
\end{tabular}

(C) Universidad San Ignacio de Loyola, Vicerrectorado de Investigación, 2017.

(cc) BY-NC-ND Este artículo se distribuye bajo licencia CC BY-NC-ND 4.0 Internacional (http://creativecommons.org/licenses/by-nc-nd/4.0/). 


\section{Resumen}

El presente trabajo de investigación tiene como propósito conocer la relación existente entre las variables: Gestión pedagógica docente y ejecución instrumental en estudiantes de una Escuela Superior de Formación Artística. Es una investigación de nivel descriptivo - correlacional que busca hallar la relación que existe entre ambas variables. La muestra del estudio estuvo conformada por 30 estudiantes de la banda sinfónica a quienes se le aplicó dos instrumentos: Cuestionario para la gestión pedagógica y una Lista de cotejo para la ejecución instrumental en la banda sinfónica. Los resultados de la investigación evidenciaron al aplicar la fórmula de correlación de Spearman un nivel de significancia del 5\%, con una correlación significativa de Rho = 0.843; por lo cual se determina que a muy buena gestión pedagógica de los docentes se produce muy buena ejecución instrumental de la banda sinfónica en la población en estudio. De manera específica al analizar los resultados de la competencia cognitiva y procedimental del docente en relación con la ejecución instrumental se encontró una correlación moderada, en la competencia actitudinal y didáctica del docente en relación con la ejecución instrumental se encontró un nivel de correlación buena. Concluyendo que sí existe una correlación significativa entre las variables gestión pedagógica docente y ejecución instrumental.

Palabras claves: Gestión pedagógica docente, ejecución instrumental, competencia cognitiva, competencia procedimental, competencia actitudinal, y competencia didáctica.

\section{Summary}

This research aims to know the relationship between the variables teachers' pedagogical management and instrumental performance in students from an Artistic Higher Education School. It is a descriptive and correlational research that seeks to find the relationship between both variables. The sample of the study consisted of 30 students of the symphonic band to whom two 
instruments were applied: a questionnaire for pedagogical management and a checklist for instrumental performance in the symphonic band. The results of the research showed when applying the Spearman's correlation formula a significance level of $5 \%$, with a significant correlation of Rho $=0.843$. Based on that, it is determined that a very good pedagogical management of teachers produces a very good instrumental performance of the symphonic band in the population under study. Specifically, when analyzing the results of the specific cognitive and procedural competences of the teacher in relation to the instrumental performance, a moderate correlation was found; when analyzing attitudinal and teaching competences of the teacher in relation to the instrumental performance, a good correlation was found. It was concluded that there is a significant correlation between the variables teachers' pedagogical management and instrumental performance.

Keywords: Teachers' pedagogical management, instrumental performance, cognitive competence, procedural competence, attitudinal competence and teaching competence. 
La educación actualmente está relacionada con el encargo social de transformar a los individuos y a la sociedad para beneficio de todos, pero para lograrse, el docente debe presentar una serie de características o competencias que le permitan prestar ese servicio orientado a lograr el objetivo planteado, en este caso la transformación del individuo en un profesional exitoso capaz de conducir y gestionar una banda sinfónica.

No hay que perder de vista que un docente es un profesional cuyo trabajo se encuentra orientado a resolver una necesidad especifica de la sociedad en un determinado momento y que proporciona a sus usuarios un servicio, el cual debe ser de calidad para asegurar la formación integral de los estudiantes peruanos en general y de los estudiante de una Escuela Superior de Formación Artística.

Jiménez (2013) reveló como influye la emoción en la relación enseñanza aprendizaje de un instrumento musical en la orquesta juvenil. En las conclusiones de esta investigación se destaca que a través de la relación docente - alumno, la emoción es el principal factor que genera el clima del aula, que a su vez repercute positiva o negativamente en el aprendizaje. Asimismo agrega que un clima emocional desfavorable en el que el miedo, la inseguridad y la rabia son preponderantes, no permiten que los alumnos respondan como se espera, ni que los profesores entreguen sus conocimientos de manera adecuada, perpetuando con una comunicación violenta el clima emocional desfavorable.

De igual forma, Salinas (2014) determinó la relación que existe entre la calidad de la Gestión pedagógica y la práctica docente en el nivel secundario, asimismo buscó establecer la relación que se da entre las estrategias metodológicas y didácticas con la práctica docente. En las conclusiones se destaca la existencia de una relación significativa entre la calidad de la gestión pedagógica con la práctica docente, por lo cual el investigador recomienda que es necesario que la población elabore una guía metodológica para evaluar la calidad de la gestión pedagógica y medir la práctica docente en el aula, considerando los criterios e indicadores de evaluación que se cuenta en el marco del buen desempeño docente propuesto por el Ministerio de Educación. 
La presente investigación tiene como objeto conocer la relación entre Gestión pedagógica docente y ejecución instrumental en estudiantes de una Escuela Superior de Formación Artística. Resaltando que la gestión pedagógica en los diferentes niveles de enseñanza superior y en las distintas carreras profesionales adquiere vital importancia para solucionar los problemas que enfrenta el proceso educativo en la formación de los profesionales, tomando en consideración las tendencias actuales y el énfasis en la intervención cada vez más creciente y activa de los estudiantes en su formación.

Una adecuada gestión pedagógica docente favorece el logro de los aprendizajes y la adquisión de capacidades y competencias necesarias en los estudiantes, los cuáles se evidenciaran en su desempeño académico y futuro desarrollo profesional. Basándonos en las distintitas competencias que implica la gestión pedagógica, tenemos en cuenta: la competencia cognitiva, competencia procedimental, competencia actitudinal y competencia didáctica. Las cuales juegan un rol importante al momento de asimilar y adquirir un nuevo aprendizaje para la vida. De esta manera se ha visto necesario la investigación de la gestión pedagógica docente en relación con la ejecución instrumental de la banda sinfónica, para conocer la implicancia que tiene la primera variable sobre esta última. Reconociendo que la ejecución instrumental es una habilidad o destreza práctica que debe ser aprendido por los estudiantes y será demostrado de forma directa en la puesta en escena, al tocar los distintos instrumentos musicales.

El aporte del presente estudio permitirá conocer la relación existente entre la variable gestión pedagógica docente y la variable ejecución instrumental en la banda sinfónica, de manera que sirvan en las futuras investigaciones de este tipo, con la finalidad de mejorar los aprendizajes prácticos adquiridos, en el área de la ejecución instrumental. Además, teniendo en cuenta la mejora continúa de la gestión pedagógica para alcanzar una formación profesional de calidad. 


\section{Gestión pedagógica docente.}

Una de las variables considerada por diferentes autores es la gestión pedagógica, según Rey (2014) la calidad de la educación involucra la calidad académica y/o la calidad pedagógica, entendida como un nexo entre el pensamiento pedagógico, el ejercicio de la enseñanza y la generación de nuevos conocimientos y de nuevas maneras de responder a sus responsabilidades sociales, culturales y éticas en la formación integral del alumno. Entonces, la calidad educativa en la educación se mide por la calidad del proceso de enseñanza aprendizaje desarrollado entre docente y estudiante a lo largo de su estadía en la institución; así mismo, será óptimo tal proceso cuando el perfil del egresado sea óptimo.

De acuerdo con Montas (2008) es acompañar y facilitar a los estudiantes en el proceso de enseñanza aprendizaje para propiciar situaciones que favorecen la elaboración de nuevos saberes y el desarrollo de los valores y las actividades previstas en el nuevo currículum. La gestión pedagógica es un instrumento de acción eficaz para que el trabajo en equipo y el proyecto de escuela sean los principales receptores de la práctica didáctica de aula y de la formación continua de los docentes Espinel (2002),

\section{Ejecución instrumental en la banda sinfónica.}

Sevilla (2016) define a la ejecución instrumental como la acción de tocar una melodía, a través de un instrumento musical de cuerda, percusión o viento. Para ejecutar necesitas algunos requisitos: Aproximarte al instrumento, jugar con el aprender, las notas, como suena, realizar ejercicios básicos con el instrumento que elegiste. Leal (2010) la ejecución instrumental es interpretar cualquier forma musical en diferentes escenarios nacionales e internacionales en un determinado instrumento musical académico o popular.

Aiello (2012) es una forma de expresarse a través de la ejecución de instrumentos musicales, de tal manera que se desarrollen y trabajen en el 
estudiante diversas capacidades a la vez realizar una coordinación audio-visomotora, logrando valores como el respeto por el otro, una actividad colaborativa que tiene como fruto el ensamble instrumental.

La ejecución instrumental se dimensiona en los siguientes: Lectura musical, dominio de la teoría musical, dominio de las técnicas de ejecución y ensamble en grupo.

\section{Método}

El diseño de investigación que se utilizó fue descriptivo - correlacional.

\section{Población y muestra}

La población estuvo constituida por 150 estudiantes de la Escuela Superior de Formación Artística Pública "Condocunca" - Ayacucho, matriculados en los diferentes ciclos durante el semestre académico 2015 - II. El muestreo fue aleatorio, y debido a la disponibilidad en los cursos, se obtuvo como muestra 30 estudiantes integrantes de la banda sinfónica.

\section{Instrumentos de recolección de datos.}

La recolección de información, fue obtenida mediante la aplicación de dos instrumentos:

El cuestionario para la variable gestión pedagógica docente está constituido por 20 ítems de tipo abierto, es decir, escala Likert que brindan información acerca de variable Gestión pedagógica docente a través de la evaluación de cuatro dimensiones: Competencia cognitiva, procedimental, actitudinal y didáctica. Las respuestas se califican: de acuerdo a siguiente escala de valoración y el índice correspondiente. Nunca (1), casi nunca (2), a veces (3), casi siempre, (4) y siempre (5). 
Por otro lado la lista de cotejo para la variable ejecución instrumental de la banda sinfónica, está constituido por 19 ítems de tipo abierto, escala Likert que brindan información acerca del variable desarrollo de los factores físicos consistente en cuatro dimensiones: lectura musical, dominio de la teoría musical, dominio de las técnicas de ejecución y el ensamble en grupo. Las respuestas se califican: de acuerdo a siguiente escala de valoración y el índice correspondiente. Pésimo (1), malo (2), regular (3), bueno (4) у muy bueno (5).

Ambos instrumentos recibieron la validez, a través del criterio de expertos, el instrumento de Gestión pedagógica docente y el de Ejecución instrumental obtuvieron una valoración de 90\%, respectivamente. De igual forma, ambos instrumentos demostraron ser confiables el alfa de Cronbach fue:

\section{Tabla 1.}

Confiabilidad de instrumentos de investigación.

\begin{tabular}{|c|c|}
\hline Instrumentos de investigación & Alfa de Cronbach \\
\hline Gestión Pedagógica Docente & 0.806 \\
\hline $\begin{array}{c}\text { Ejecución Instrumental en la } \\
\text { Banda Sinfónica }\end{array}$ & 0.955 \\
\hline
\end{tabular}

\section{Plan de análisis.}

Para el tratamiento estadístico, se empleó el paquete computacional SPSS versión 21. Primero se aplicó un cuestionario para la variable gestión pedagógica docente en el auditorio de la institución lugar en donde se desarrolla habitualmente el ensayo del Taller de la Banda Sinfónica de la institución, en el día y hora acordado con el director, todos los integrantes de la Banda Sinfónica participaron activamente. Antes del llenado del cuestionario se aclaró a los estudiantes sobre el objetivo del estudio y el empleo de la información, posteriormente firmaron la carta de consentimiento informado, 
para luego iniciar con el llenado del cuestionario. Segundo se aplicó una lista de cotejo para la Variable ejecución instrumental, a todos los integrantes de la Banda Sinfónica en los diferentes escenarios en donde se desarrollaron los ensayos y las presentaciones musicales del Taller.

\section{Resultados}

\section{Análisis descriptivo.}

\section{Tabla 2.}

Relación entre las respuestas de las variables: Gestión pedagógica docente y ejecución instrumental de la banda sinfónica.

\begin{tabular}{|c|c|c|c|}
\hline \multicolumn{3}{|c|}{ Correlaciones } & \multirow{2}{*}{$\begin{array}{l}\text { Ejecución Instrumental } \\
\text { de la Banda Sinfónica }\end{array}$} \\
\hline \multirow{7}{*}{$\begin{array}{l}\text { Rho de } \\
\text { Spearman }\end{array}$} & \multirow{3}{*}{$\begin{array}{l}\text { Gestión Pedagógica } \\
\text { Docente }\end{array}$} & $\begin{array}{l}\text { Coeficiente de } \\
\text { correlación }\end{array}$ & \\
\hline & & Sig. (bilateral) & .000 \\
\hline & & $\mathrm{N}$ & 30 \\
\hline & \multirow{4}{*}{$\begin{array}{l}\text { Ejecución } \\
\text { Instrumental de la } \\
\text { Banda Sinfónica }\end{array}$} & $\begin{array}{l}\text { Coeficiente de correl- } \\
\text { ación }\end{array}$ & 1.000 \\
\hline & & & \\
\hline & & Sig. (bilateral) & .00 \\
\hline & & $\mathrm{N}$ & 30 \\
\hline
\end{tabular}

Al aplicar la fórmula de Rho de Spearman, el coeficiente de correlación resultó de 0.843 y la significancia de $p_{\text {_ }}$ valor $=.000$, por lo tanto se establece un nivel de correlación significativa muy buena. 


\section{Tabla 3.}

Relación entre las respuestas de la variable Competencia cognitiva y la ejecución instrumental de la banda sinfónica.

\begin{tabular}{|c|c|c|c|}
\hline & \multicolumn{2}{|c|}{ Correlaciones } & $\begin{array}{c}\text { Ejecución } \\
\text { Instrumental de la } \\
\text { Banda Sinfónica }\end{array}$ \\
\hline \multirow{6}{*}{ Rho de Spearman } & \multirow{3}{*}{$\begin{array}{l}\text { Competencia } \\
\text { cognitiva }\end{array}$} & $\begin{array}{l}\text { Coeficiente de cor- } \\
\text { relación }\end{array}$ & 0.416 \\
\hline & & Sig. (bilateral) & .022 \\
\hline & & $\mathrm{N}$ & 30 \\
\hline & \multirow{3}{*}{$\begin{array}{l}\text { Ejecución I } \\
\text { nstrumental de la } \\
\text { Banda Sinfónica }\end{array}$} & $\begin{array}{l}\text { Coeficiente de } \\
\text { correlación }\end{array}$ & 1.000 \\
\hline & & Sig. (bilateral) & 0.0 \\
\hline & & $\mathrm{N}$ & 30 \\
\hline
\end{tabular}

Al aplicar la fórmula de Rho de Spearman, el coeficiente de correlación resultó 0.416 y la significancia de $\mathrm{p}_{-}$valor $=.022$, por lo tanto se establece un nivel de correlación significativa moderada.

\section{Tabla 4.}

Relación entre las respuestas de la variable: Competencia procedimental y ejecución instrumental de la banda sinfónica.

\begin{tabular}{|c|c|c|c|}
\hline & Correlaciones & & $\begin{array}{l}\text { Ejecución Instrumental } \\
\text { de la Banda Sinfónica }\end{array}$ \\
\hline \multirow{7}{*}{ Rho de Spearman } & \multirow[t]{3}{*}{$\begin{array}{l}\text { Competencia } \\
\text { procedimental }\end{array}$} & $\begin{array}{l}\text { Coeficiente de } \\
\text { correlación }\end{array}$ & 0.464 \\
\hline & & Sig. (bilateral) & .010 \\
\hline & & $\mathrm{N}$ & 30 \\
\hline & \multirow{4}{*}{$\begin{array}{l}\text { Ejecución } \\
\text { Instrumental de la } \\
\text { Banda Sinfónica }\end{array}$} & Coeficiente de & 1.000 \\
\hline & & correlación & \\
\hline & & Sig. (bilateral) & 0.0 \\
\hline & & $\mathrm{N}$ & 30 \\
\hline
\end{tabular}

Al aplicar la fórmula de Rho de Spearman, el coeficiente de correlación resultó de 0.464 y la significancia de $p_{\text {_ }}$ valor $=.010$, por lo tanto se establece un nivel de correlación significativa moderada. 


\section{Tabla 5.}

Relación entre las respuestas de la variable: Competencia actitudinal y ejecución instrumental de la banda sinfónica.

\begin{tabular}{|c|c|c|c|}
\hline & Correlaciones & $\begin{array}{l}\text { Ejecución } \\
\text { Instrumental de la Banda Sinfónica }\end{array}$ & \\
\hline \multirow{7}{*}{ Rho de Spearman } & \multirow{3}{*}{$\begin{array}{l}\text { Competencia } \\
\text { actitudinal }\end{array}$} & $\begin{array}{l}\text { Coeficiente de } \\
\text { correlación }\end{array}$ & 0.654 \\
\hline & & Sig. (bilateral) & .000 \\
\hline & & $\mathrm{N}$ & 30 \\
\hline & \multirow{4}{*}{$\begin{array}{l}\text { Ejecución } \\
\text { Instrumental } \\
\text { de la Banda } \\
\text { Sinfónica }\end{array}$} & & 1.000 \\
\hline & & $\begin{array}{l}\text { Coeficiente de } \\
\text { correlación }\end{array}$ & \\
\hline & & Sig. (bilateral) & 0.0 \\
\hline & & $\mathrm{N}$ & 30 \\
\hline
\end{tabular}

Al aplicar la fórmula de Rho de Spearman, el coeficiente de correlación resultó 0.654 y la significancia de $p_{\text {_ }}$ valor $=0.000$, por lo tanto se establece un nivel de correlación significativa buena.

\section{Tabla 6.}

Relación entre las respuestas de la variable: Competencia didáctica con la dimensión ejecución instrumental en la banda sinfónica.

\begin{tabular}{|c|c|c|c|}
\hline & Correlaciones & & $\begin{array}{c}\text { Ejecución } \\
\text { Instrumental de la } \\
\text { Banda Sinfónica }\end{array}$ \\
\hline \multirow{6}{*}{ Rho de Spearman } & \multirow{3}{*}{ Competencia didáctica } & $\begin{array}{l}\text { Coeficiente de } \\
\text { correlación }\end{array}$ & 0.736 \\
\hline & & Sig. (bilateral) & 0.000 \\
\hline & & $\mathrm{N}$ & 30 \\
\hline & \multirow[t]{3}{*}{$\begin{array}{l}\text { Ejecución Instrumental } \\
\text { de la Banda Sinfónica }\end{array}$} & $\begin{array}{l}\text { Coeficiente de } \\
\text { correlación }\end{array}$ & 1.000 \\
\hline & & Sig. (bilateral) & 0.0 \\
\hline & & $\mathrm{N}$ & 30 \\
\hline
\end{tabular}


Al aplicar la fórmula de Rho de Spearman, el coeficiente de correlación resultó de 0.736 y la significancia de $\mathrm{p}_{-}$valor $=0.000$, por lo tanto se establece un nivel de correlación significativa buena.

\section{Discusión}

Al analizarse la correlación entre la variables gestión pedagógica docente con la ejecución instrumental de la banda sinfónica se encontró un coeficiente de correlación muy buena de $\mathrm{Rho}=0.843$ entre ambas variables. Determinándose que a buena gestión pedagógica de los docentes se produce buena ejecución instrumental de la banda sinfónica. Logrando así, los objetivos de aprendizaje por parte de los estudiantes.

Al respecto, Montas (2008) opina que la gestión pedagógica es acompañar y facilitar a los estudiantes en el proceso de enseñanza aprendizaje para propiciar situaciones que favorecen la elaboración de nuevos saberes y el desarrollo de los valores y las actividades previstas en el nuevo currículum.

Por otro lado, se halló un nivel de correlación moderada de Rho $=0.416$ entre la dimensión Competencia cognitiva con relación a la variable la ejecución instrumental de la banda sinfónica. Determinándose que a moderada competencia cognitiva se produce una moderada ejecución instrumental de la banda sinfónica. Desprendiéndose de este resultado que los docentes necesitan dominar mejor los conocimientos y habilidades propios de la materia que imparten.

Vázquez (2007) señala que las competencias cognitivas del profesor pueden referirse principalmente a los siguientes ámbitos del ejercicio profesional: espacio de la relación con cada alumno y con ellos en común, espacio de relación con otros profesores, directivos y otros profesionales, espacio de relación con familia y la comunidad y, por último, ámbito de relación consigo mismo.

Con relación a la competencia procedimental y la ejecución instrumental de la banda sinfónica resulto un coeficiente de correlación de Rho $=0.464$. 
Por lo tanto, se establece un nivel de correlación significativa moderada. Se determina como el caso anterior, que a moderada competencia procedimental se produce moderada ejecución instrumental de la banda sinfónica. Considerando por dicha razón, que la gestión pedagógica docente llevada a cabo debe incidir más en el aprendizaje práctico y dominio de estrategias, técnicas, habilidades, y métodos que se transfieran al alumno con facilidad para llevar a cabo la ejecución instrumental.

Para Tobón (2006) el saber hacer consiste en saber actuar con respecto a la realización de una actividad o la resolución de un problema, comprendiendo el contexto y teniendo como base la planeación. De hecho, al hablar de estructurar el desempeño idóneo en los últimos años han surgido dos tendencias: la primera consiste en enfatizar el aprendizaje y en la persona que aprende, surgiendo una serie de conceptos como enseñar a pensar, aprender a aprender entre otros; cuyo centro es el aprendizaje descuidando la actuación, y la segunda, enfatiza en el desempeño y no en el saber; donde lo central es la eficiencia y eficacia, importando los resultados evaluables públicamente, dejando de lado el procesamiento de la información.

Referente a la competencia actitudinal y la ejecución instrumental de la banda sinfónica, se encontró un nivel de correlación significativa buena, de Rho $=0.654$. Determinándose que a buena competencia actitudinal se produce buena ejecución instrumental de la banda sinfónica. Se evidencia a través de dicho resultado el buen manejo de las habilidades sociales en la gestión pedagógica docente para desarrollar predisposición y motivación, entre otros factores positivos presentes en los estudiantes en la ejecución instrumental.

Pinto (2011) refiere que esta competencia está relacionada con los aspectos éticos que orientan y otorgan sentido al saber y al hacer. Sin embargo, la formación por competencias no se limita a una formación orientada exclusivamente hacia el hacer, sino también del saber-hacer, en consecuencia, lo teórico, lo procedimental y actitudinal deben estar armónicamente considerados en los procesos de enseñanza-aprendizaje. 
Por último, la competencia didáctica con relación a la ejecución instrumental de la banda sinfónica se encontró una nivel de correlación significativa buena de $\mathrm{Rho}=0.736$. Determinándose que a buena competencia didáctica se produce muy buena ejecución instrumental de la banda sinfónica. En consecuencia, se evidencia que las habilidades didácticas docentes aplicadas en clases favorecen a lograr el aprendizaje deseado en la ejecución instrumental de la banda sinfónica.

Toledo (2006) refiere que la competencia didáctica es la habilidad del docente para establecer una relación comprensible, esto significa el construir un conjunto de relaciones sociales entre él y sus estudiantes en la perspectiva de realizar una acción delimitada en un cuadro espacio-temporal académico determinado cuya finalidad es provocar cambios en ellos. Dicho de otra forma, cómo identifica y transforma el saber de referencia, los contenidos, la información, en agentes (medios) de cambios para sus estudiantes, en saber aprendido.

\section{Referencias}

Aiello, R. (2012). Ejecución Instrumental [miaulavirtualdemusica.com]. Recuperado de: https://sites.google.com/site/miaulavirtualdemusica/ home/ejecucion-instrumental

Espinel, A. (2002). Abordando los espacios escolares. Argentina: Editorial Lumex.

Finol de Franco, M., Vallejo, R., \& García de Hurtado, M. C. (2012). Gestión directiva en el marco de la evaluación institucional en organizaciones educativas. Multiciencias, 12, 88-93.

Jiménez, M. (2013). Interpretación y vínculo en una orquesta juvenil (Tesis de maestría).

Universidad Academia Humanismo Cristiano, Santiago.

Leal, P. (2010). Ejecucion Instrumental: Explicacion del Nuevo Curriculo de UNEARTE [Diapositiva]. Venezuela: UNEARTE. 
Mejía, E. (2014). Metodología de la Investigación. Cuantitativa - cualitativa y redacción de la Tesis. Bogotá: Ediciones de la Universidad de Bogotá.

Montas, A. (2008).Gestión Pedagógica Docente (Texto inédito).República Dominicana.

Pinto, H. (2011). Formación de competencias docentes en matemática de educación básica. Cuadernos de Educación y Desarrollo, 3(26). Recuperado de: http://www.eumed.net/rev/ced/26/hp.htm

Rey, A. (2014). Gestión pedagógica del docente y dimensión axilógica del conocimiento. Dialógica, 11(1), 111-132.

Sevilla, C. (2016). Ejecución instrumental en la Banda Sinfónica (Texto inédito).

Sandoval, A. (2008). Competencias del docente para la aplicación del eje transversal ambiente (Tesis de Maestría). Universidad de Zulia, Marcaibo.

Salinas, E. (2014). La calidad de la gestión pedagógica y su relación con la práctica docente en el nivel secundaria de la Institución Educativa Policía Nacional del Perú “Juan Linares Rojas”, Oquendo, Callao (Tesis de Maestría). Universidad Nacional Mayor de San Marcos, Lima. Sloboda, J. (2005). Experimental studies of music reading: a review. En J. A. Sloboda (Ed.),

Exploring the musical mind (pp.27-42). New York: Oxford University Press.

Tobón (2006). Competencias, calidad y educación superior ( $\left(1^{\mathrm{a}}\right.$ Ed).Bogotá: Cooperativa Editorial Magisterio.

Toledo, P.M. (2006). Competencias didácticas, evaluativas y metacognitivas. Revista de Orientación Educacional, 20(38), 105-116.

Vázquez, G. (2007). La formación de la competencia cognitiva del profesor. Estudios sobre Educación, 12, 41-57. Recuperado de: http://dadun.unav. edu/bitstream/10171/8995/1/12\%20Estudios\%20Ec.pdf 


\section{Anexo A: Cuestionario de la variable Gestión pedagógica docente}

\section{Instrucciones}

Estimado alumno marque con un aspa(x), la respuesta que Ud. crea conveniente en uno de los recuadros.

\begin{tabular}{|c|c|c|c|c|c|c|}
\hline \multicolumn{2}{|r|}{ VARIABLE: GESTION PEDAGOGICA DOCENTE } & \multirow{2}{*}{ 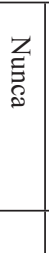 } & \multirow{2}{*}{ 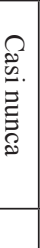 } & \multirow[t]{2}{*}{ 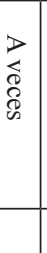 } & \multirow{2}{*}{ 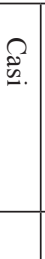 } & \multirow{2}{*}{ 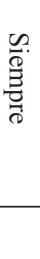 } \\
\hline $\mathrm{CO}$ & PETENCIA COGNITIVA & & & & & \\
\hline 1 & El docente facilita información a los alumnos sobre la teoría musical & & & & & \\
\hline 2 & El docente realiza la práctica de la lectura musical con sus alumnos & & & & & \\
\hline 3 & El docente asesora a sus alumnos en la ejecución instrumental & & & & & \\
\hline 4 & El docente conoce las técnicas de ejecución instrumental & & & & & \\
\hline 5 & $\begin{array}{l}\text { El docente gestiona viajes a festivales y/o capacitaciones de } \\
\text { música desarrollados en otras regiones }\end{array}$ & & & & & \\
\hline 6 & $\begin{array}{l}\text { El docente realiza sesiones de aprendizaje individualizadas para el } \\
\text { aprendizaje de la ejecución instrumental para los integrantes de la } \\
\text { Banda Sinfónica. }\end{array}$ & & & & & \\
\hline \multicolumn{2}{|c|}{ COMPETENCIA PROCEDIMENTAL } & & & & & \\
\hline 7 & El docente demuestra domino de la dirección musical & & & & & \\
\hline 8 & El docente demuestra autoridad en la dirección musical & & & & & \\
\hline 9 & $\begin{array}{l}\text { El docente gestiona la ejecución instrumental con precisión en sus } \\
\text { alumnos integrantes de la Banda Sinfónica }\end{array}$ & & & & & \\
\hline 10 & $\begin{array}{l}\text { El docente gestiona en los alumnos la ejecución de otros } \\
\text { instrumentos musicales a parte de su instrumento principal para } \\
\text { integrar la banda } \\
\text { sinfónica. }\end{array}$ & & & & & \\
\hline \multicolumn{2}{|c|}{ COMPETENCIA ACTITUDINAL } & & & & & \\
\hline 11 & $\begin{array}{l}\text { El docente negocia con profesores de otras asignaturas para motivar } \\
\text { a los integrantes de la Banda Sinfónica. }\end{array}$ & & & & & \\
\hline 12 & $\begin{array}{l}\text { El docente impulsa en los integrantes de la Banda Sinfónica el } \\
\text { deseo de hacer las cosas bien. }\end{array}$ & & & & & \\
\hline 13 & El docente gestiona iniciativas para mejor la ejecución instrumental. & & & & & \\
\hline 14 & $\begin{array}{l}\text { El docente gestiona una actitud de superación y la práctica de los } \\
\text { valores dentro de los integrantes de la Banda Sinfónica. }\end{array}$ & & & & & \\
\hline
\end{tabular}




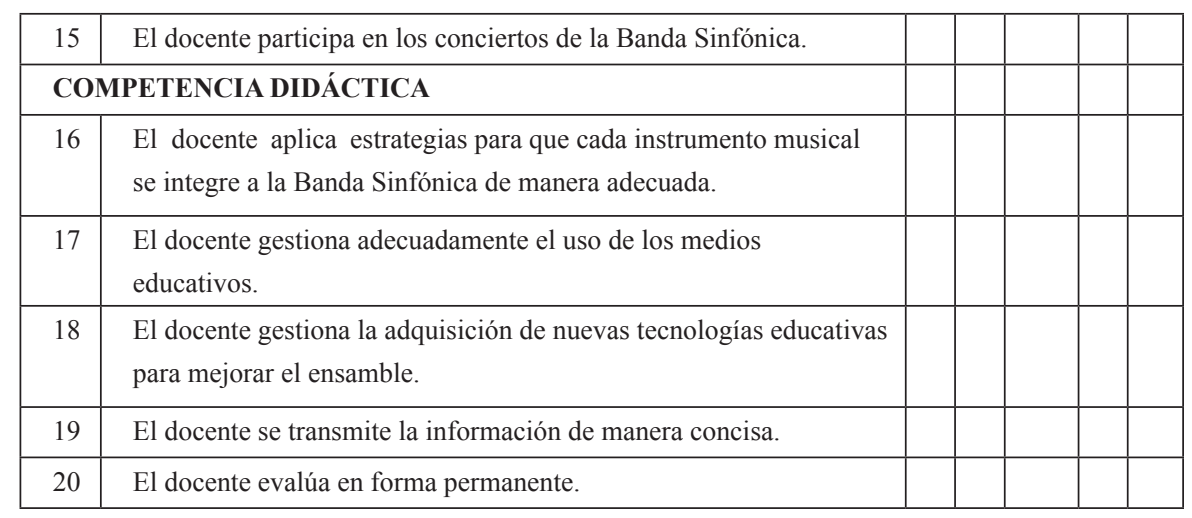




\section{Anexo B: Lista de cotejo de la variable: Ejecucion instrumental}

Estudiante Instrumento:

INDICACIONES: El investigador tiene la intención de recoger información relacionada a la ejecución instrumental en la banda sinfónica de los estudiantes de la institución. Es menester señalar que, las informaciones que se recogerán solo sirven para aspectos investigativos en el marco de la ejecución del estudio.

Marque con aspa (X) donde corresponda.

\section{ESCALA DE VALORACIÓN:}

Muy bueno $=\mathbf{5}$ Bueno $=4$ Regular $=\mathbf{3}$ Malo $=\mathbf{2}$ Pésimo $=\mathbf{1}$

\begin{tabular}{|c|c|c|c|c|c|}
\hline \multirow[t]{2}{*}{ ITEMS } & \multicolumn{5}{|c|}{$\begin{array}{c}\text { ESCALA DE } \\
\text { VALOACIÓN }\end{array}$} \\
\hline & 5 & 4 & 3 & 2 & 1 \\
\hline 1. Lee música en pentagrama a primera vista & & & & & \\
\hline 2. Demuestra dominio del solfeo hablado & & & & & \\
\hline 3. Demuestra dominio del solfeo cantado & & & & & \\
\hline 4. Entona las melodías que se interpreta en la Banda sinfónica & & & & & \\
\hline 5. interpreta la línea melódica teniendo en cuenta el contexto histórico de la obra & & & & & \\
\hline 6. Es capaz de seguir la secuencia de la línea melódica & & & & & \\
\hline 7. Maneja distintos ritmos y los adecua a la necesidades de la Banda Sinfónica & & & & & \\
\hline 8. Maneja el ritmo interno sin necesidad de utilizar metrónomo & & & & & \\
\hline 9. Se adecua fácilmente al trabajo armónico de la Banda Sinfónica & & & & & \\
\hline 10. Conoce el circulo armónico por intervalos de quintas en su instrumento & & & & & \\
\hline 11. El sonido de su instrumento es adecuado para la banda sinfónica & & & & & \\
\hline 12. Utiliza una técnica de respiración adecuada para la interpretación de pasajes largos & & & & & \\
\hline 13. Utiliza una técnica correcta de articulación y fraseo en una idea musical & & & & & \\
\hline 14. Maneja una digitación exacta en el instrumento musical que interpreta & & & & & \\
\hline 15. Maneja una postura correcta para la ejecución de su instrumento musical & & & & & \\
\hline 16. Demuestra dominio del instrumento como solista & & & & & \\
\hline 17. Demuestra capacidad para interpretar su instrumento musical de manera grupal & & & & & \\
\hline 18. El instrumento musical que ejecuta está debidamente afinado en 4:40 & & & & & \\
\hline 19. Posee las competencias necesarias para desenvolverse dentro de unabanda sinfónica. & & & & & \\
\hline 20. Participa activamente en los conciertos de la banda sinfónica & & & & & \\
\hline
\end{tabular}

